\title{
LOS MATERIALES DE CONCHA UTILIZADOS EN LA MANUFACTURA DE ATAVÍOS EN LAS OFRENDAS DEL TEMPLO MAYOR DE TENOCHTITLAN
}

\section{THE SHELL MATERIALS USED IN THE MANUFACTURE OFQRNAMENTS IN THE OFFERINGS OF THE TEMPLO MAYOR OF TENOCHTITLAN}

\author{
Belem Zúñiga-Arellano ${ }^{1}$ \\ belem_zu@yahoo.com' Orcid 0000-0003-1136-2325 \\ Alejandra Aguirre Molina ${ }^{1}$ \\ cocijo30@yahoo.com' Orcid 0000-0003-1915-1389 \\ Israel Elizalde Mendez ${ }^{1}$ \\ elizalde-israel@hotmail.com / Orcid 0000-0003-0596-7301

\section{RESUMEN}

Como parte de las excavaciones del Proyecto Templo Mayor en el centro de la Ciudad de México, entre 2008 y 2013 fueron recuperados cientos de artefactos elaborados con caracoles y conchas. En este texto estudiamos 479 objetos de concha procedentes de cuatro ofrendas que fueron utilizados para la elaboración de atavíos empleados para ornamentar 46 cuchillos de pedernal y, a una loba mexicana (Canis lupus baileyi). Se trabajó desde tres diferentes perspectivas: biológica, arqueológica e iconográfica. Estor enfoques se integran y complementan para dar una mejor interpretación de los contextos rituales. Además, nos apoyamos en el uso de herramientas digitales como el prograna AutoCAD, para visualizar con mayor facilidad, las asociaciones contextuales que había entre los objetos depositados en las ofrendas. Finalmente, encontramos patrones distintivos entre las especies de moluscos y su uso; Un ejemplo es el uso de caracoles del género Olivella para la fabricación de pendientes.

Palabras clave: Concha; manufactura; atavios; Templo Mayor.

\footnotetext{
${ }^{1}$ Investigador, INAH, México.
}

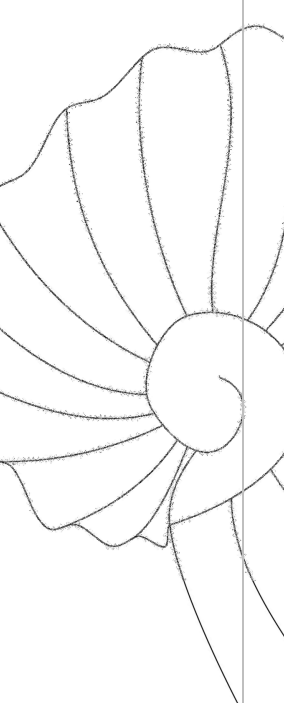




\begin{abstract}
During the excavations of the Templo Mayor Project, in the center of Mexico City, between 2008 and 2013 hundreds of shell artifacts were recovered. In this paper we studied 479 shell artifacts from four offerings that were used to make ornaments dressing 46 flint knives and a mexican wolf (Canis lupus baileyi). We worked on three different perspectives: biological, archaeological and iconographic. These approaches were integrated to give us a better interpretation about the ritual contexts. Furthermore, we applied digital tools like AutoCAD to visualize the spatial relationships, which allowed us to easily distinguish the contextual associations between the objects in the offerings. We found distinctive patterns between shell specie and usage; the most distinctive case was the usage of Olivella for the manufacture of pendants.
\end{abstract}

Keywords: Keywords: shell; manufacturing, ornaments; Templo Mayor.

\title{
CONTEXTO DE LA INVESTIGACION
}

Los materiales ofrendados en el Templo Mayor y sus áreas circundantes son el reflejo del pensamiento mexica. Los cientos de miles de objetos encontrados en las inmediaciones del Huei Teocalli responden a su ideología y cosmovisión conformando complejos discursos en las ofrendas del Templo Mayor de Tenochtitlan. Los materiales malacológicos ya fueran depositados en su materia más orgánica, o bien, a manera de artefactos, buscaban trasminar un mensaje y formaban un puente de comunicación entre los hombres y las deidades. En este contexto, los artefactos de concha finamente elaborados formaron grupos 0 unidades discretas. Las excavaciones arqueológicas realizadas por el Proyecto Templo Mayor han permitido entender un poco más referente al uso y simbolismo de estos objetos.

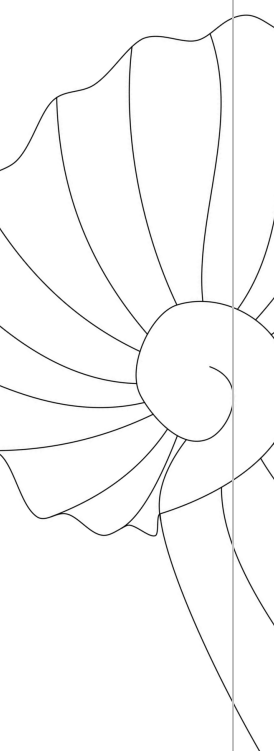


En 2007, tras el descubrimiento del monolito de la diosa terrestre Tlaltecuhtli, dio inicio la séptima temporada de excavación del Proyecto Templo Mayor, bajo la dirección del Dr. Leonardo López Luján. Entre los objetivos del proyecto se contaban la prospección, la topografía y la excavación del terreno del antiguo Mayorazgo de Nava Chávez, donde se encontró la escultura monumental (LÓPEZ y CHÁNEZ, 2010: 294-298). El sitio se localiza frente al Templo Mayor de Tenochtitlan, en el Centro Histórico de la Ciudad de México. Hasta la fecha se han descubierto 63 ofrendas, de las que se recuperaron más de 70000 elementos en un área de poco menos de $500 \mathrm{~m}^{2}$. La Plaza Oeste guarda una importante posición entorno al recinto ceremonial. Al ubicarse al frente del Templo Mayor fue el escenario de las más importantes prácticas religiosas (LÓPEZ, 2019: 53). Los materiales encontrados en este sector, sin duda destacan por su forma, tamaño o distribución.

Entre los hallazgos más importantes al momento de destapar los depósitos, se encontraron varios cuchillos de pedernal y los esqueletos de algunos animales portando diversos ornamentos elaborados con diferentes materias primas, entre ellos destacaron 546 artefactos manufacturados con las conchas de moluscos marinos y almejas de agua dulce. Estos elementos formaban parte de sus atavíos y les fueron colocados para caracterizarlos como diferentes deidades del cosmos mexica.

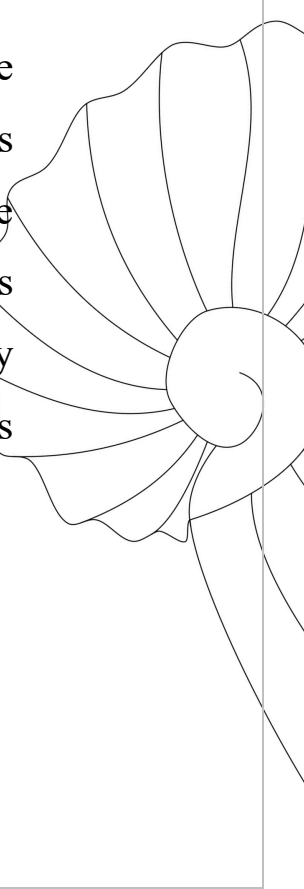


Los artefactos de concha fueron exhumados de las ofrendas 123, 125, 126 y 137, ubicadas en la Plaza Oeste del Templo Mayor, las cuatro estaban asociadas al monolito de Tlaltecuhtli. La ofrenda 126 se encontró a escasos metros bajo el monolito, mientras que los tres depósitos restantes estaban al oeste de él, en relación con una estructura en forma de pirámide invertida, la cual contenía seis ofrendas en su interior. La cronología de los depósitos corresponde al reinado de Ahuitzotl (1486-1502).

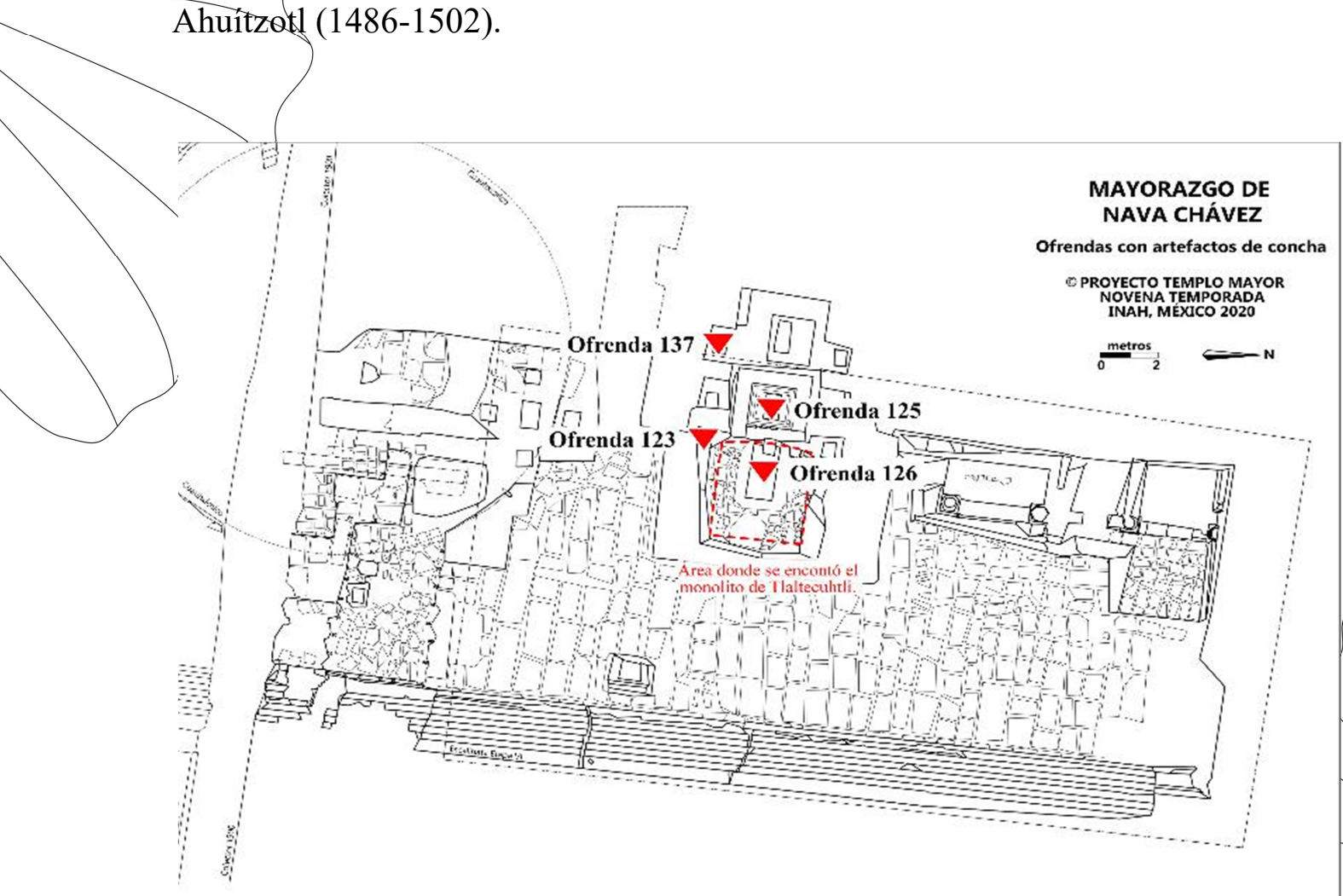

Figura 1: Las ofrendas 123, 125, 126 y 137 se ubican al frente del Templo Mayor, entorno al monolito de Tlaltecuhtli (dibujo elaborado por Michelle de Anda Rogel, cortesía PTM-INAH). 
La Ofrenda 123 se excavó en el 2008, estaba depositada en una caja hecha con sillares de tezontle, se ubicaba en la esquina sureste de una estructura en forma de piramide invertida, que simulaba un acceso al inframundo (LÓPEZ et al. 2012: 940). Se recuperaron 14 cuchillos, la mayoría estaban ataviados como cuchillos rostro y casi todos presentan una pequeña base de copal (PASCAL y GONZÁLEZ, 2009).

La Ofrenda 125 también fue descubierta en 2008, se ubicaba en el interior de la estructura en forma de pirámide invertida. Estaba contenida en una caja construida con sillares de tezontle rojo, media $85 \mathrm{~cm}$ de largo por $50 \mathrm{~cm}$ de ancho y estaba orientada en dirección este-oeste. Corresponde a uno de los depósitos más ricos en Cuanto a diversidad de especies animales y objetos (AGUIRRE y CHAVÉZ, 2010: 38).

La Ofrenda 126, al igual que la anterior fue hallada en 2008, se localizaba justo por debajo del monolito de la diosa de la tierra Tlaltecuhtli. Su continente es una caja de sillares de andesita de lamprobolita que mide $1.95 \mathrm{~cm}$ de largo, $90 \mathrm{~cm}$ de ancho y $80 \mathrm{~cm}$ de profundidad. Es uno de los depósitos rituales más grandes localizados hasta el momento. También presentaba una orientación este-oeste. Contaba con el depósito de casi 13000 elementos, entre los que sobresalían los materiales marinos y los restos óseos de animales (GONZÁLEZ et al., 2012).

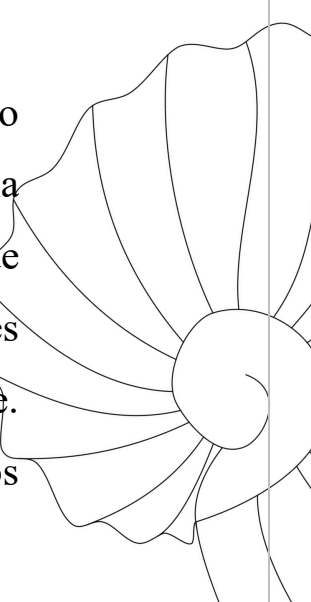


Ofrenda 137 este depósito se localizó en el 2013, en la esquina suroeste de la estructura en forma de pirámide invertida ya mencionada. Se recuperó una gran cantidad de restos de fauna como conchas, caracoles, huesos de serpiente, artefactos manufacturados con conchas, obsidiana, madera y pedernal (GARCÍA et al., 2014: 27-54).

El objetivo de este trabajo fue determinar la función y las asociaciones que los artefactos de concha guardaban dentro de los contextos de las ofrendas.

\section{METODOLOGÍA}

Los materiales se analizaron desde tres perspectivas: biológica, arqueológica e iconográfica. En primer lugar, se identificaron las especies de moluscos empleadas en la manufactura de los artefactos, se determinó su hábitat y litoral de procedencia. A continuación, con base en la clasificación tipológica de los objetos de concha propuesta por VELÁZQUEZ (1999: 33, 70-71, 99), los materiales se agruparon de acuerdo con su función en: ornamental, utilitaria y votiva.

Cabe indicar que el registro de las ofrendas del Templo Mayor resulta en suma complejo por la cantidad de materiales y su diversidad lo que en ocasiones, dificulta la comprensión del ritual.

En este caso se usó el programa AutoCAD para ubicar espacialmente cada uno de los elementos que conformaban el continente de las cuatro ofrendas analizadas. Para ello, se escanearon los dibujos de campo y se integraron las fotografías de cada uno de los niveles durante el proceso de excavación. Cada uno de los objetos

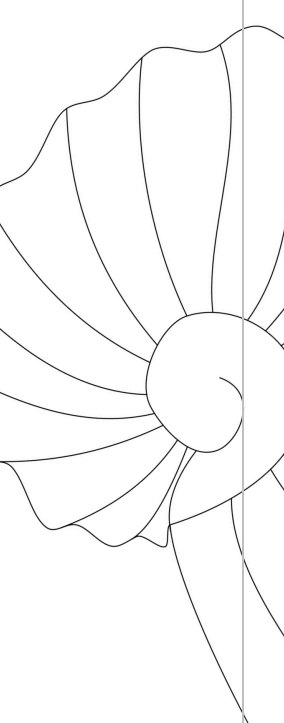


registrados en campo fue redibujado en el programa AutoCAD, a cada objeto se le asignó una clave de color. Por ejemplo, para el copal se utilizó el color amarillo y para los cuchillos se usó el color azul. En el caso de las conchas y caracoles, se les asignó un color diferente de acuerdo con su especie.

Al empalmar estos dibujos, visualizamos en conjunto todos los elementos que comprenden un discurso o unidad discreta. Para este estudio espacial partimos de la clasificación tipológica realizada con anterioridad, la profundidad de los elementos, las relaciones directas e indirectas y evaluamos los contextos de acuerdo con los principios tafonómicos (EFREMOV 1940; GIFFORD, 1981: 365368; LYMAN, 1994: 1-9; 2010: 2-4; CHÁVEZ et al., 2011: 120).

En el caso de la Ofrenda 125, que contenía más de 4000 elementos, fue necesario hacer uso del programa R Studio. Se trazaron radios de aproximación entre los objetos para identificarlos y asociarlos dentro de un área de 3 y $5 \mathrm{~cm}$ alrededor de cada objeto. Al final, el mismo sistema generó una tabla con 921 artefactos donde quedaron registrados los elementos centrales y los cinco más próximos a ellos. Con esta clase de análisis, por una parte, evitamos la subjetividad, y por otra se integraron elementos que no habían sido contemplados inicialmente.

Finalmente, los resultados contextuales obtenidos en el análisis espacial de las cuatro ofrendas se confrontaron con la información provista por las fuentes escritas y pictográficas de los siglos XV y XVI.

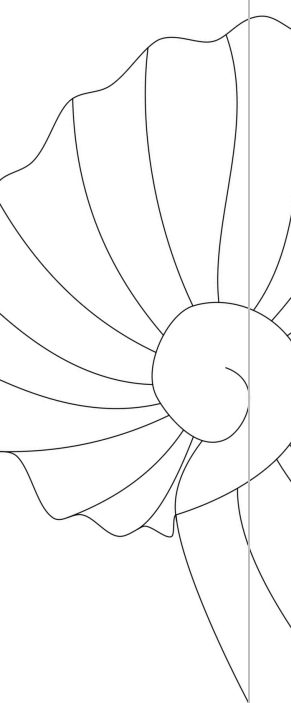




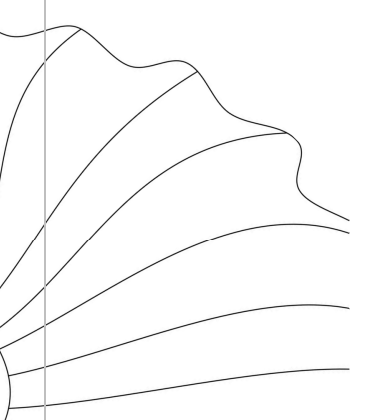

Nivel 2

Nivel 3

Nivel 4

Nivel 5

Nivel 6

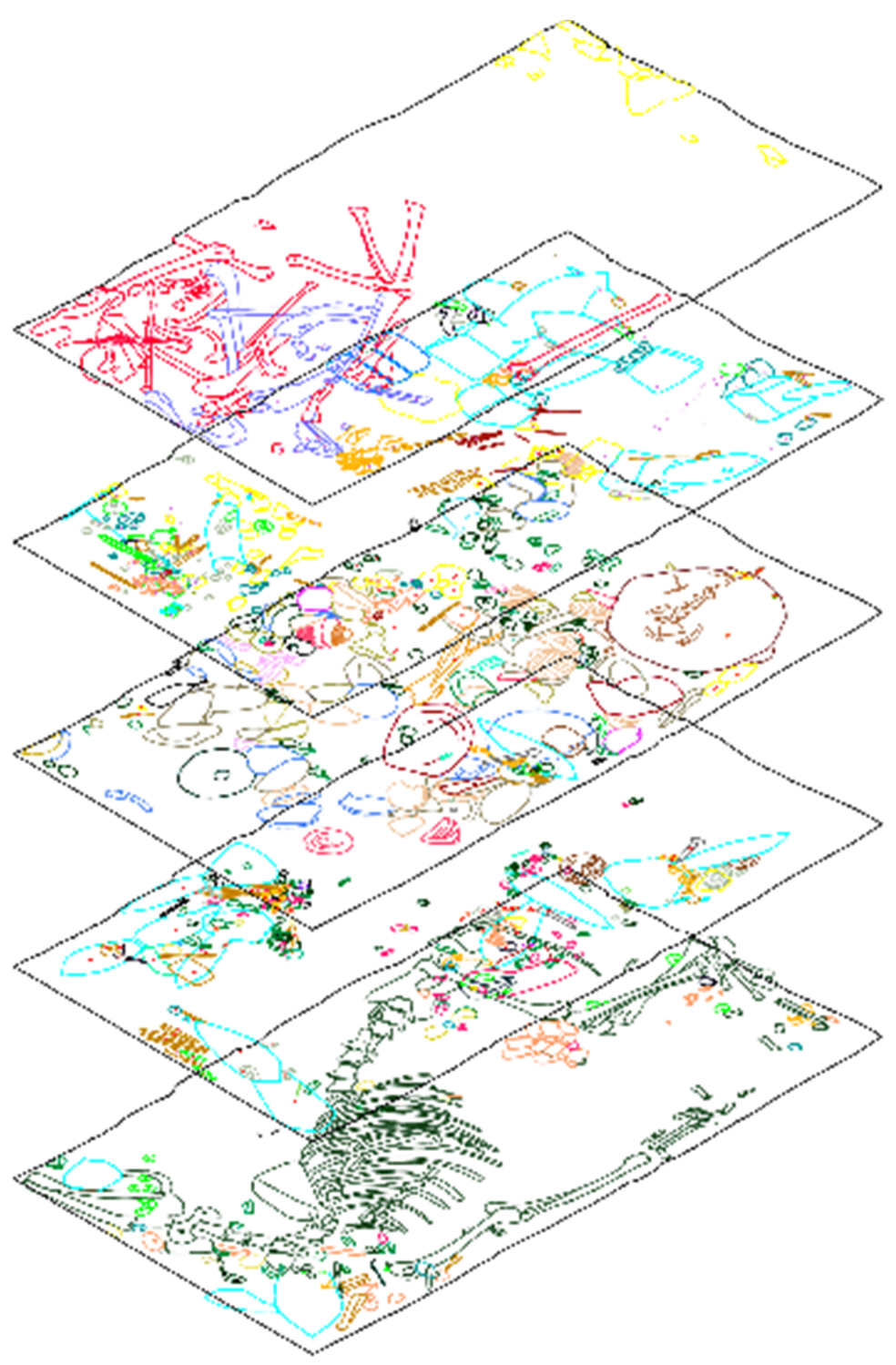

299

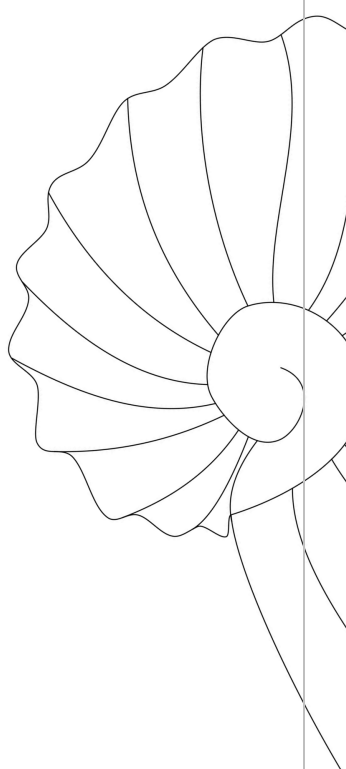

\section{(c) (i)}


Figura 2. Empalme de los dibujos de los diferentes niveles de excavación, (dibujo elaborado por Israel Elizalde, cortesía PTM-INAH).

\section{RESULTADOS}

Dentro de las cuatro ofrendas que comprenden este estudio se identificaron 546 artefactos de concha, pertenecientes a las clase Polyplacophora, conocidos comúnmente como "quitones" o "cucarachas de mar", Gastropoda, que incluye a los caracoles, liebres de mar, y babosas y Bivalvia, en la que se agrupan las almejas, mejillones, ostiones, etcétera.

\begin{tabular}{|c|c|c|c|c|c|}
\hline & \multicolumn{2}{|c|}{ Provincia malacológica } & \multirow[b]{2}{*}{$\begin{array}{c}\text { Ambas } \\
\text { provincias }\end{array}$} & \multicolumn{2}{|c|}{ Ambiente } \\
\hline V & Caribeña & Panámica & & Marino & Ríos \\
\hline 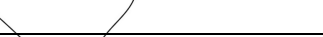 & & & & & \\
\hline \multicolumn{6}{|l|}{ Taxa identificados } \\
\hline \multicolumn{6}{|l|}{ Clase Polyplacophora } \\
\hline \multicolumn{6}{|l|}{ Familia Chitonidae } \\
\hline Chiton articulatus & & $\mathrm{x}$ & & $\mathrm{x}$ & \\
\hline \multicolumn{6}{|l|}{ Clase Gastropoda } \\
\hline \multicolumn{6}{|l|}{ Familia Neritidae } \\
\hline Nerita scabricosta & & $\mathrm{x}$ & & $\mathrm{x}$ & \\
\hline Neritina virginea & & & $\mathrm{x}$ & $\mathrm{x}$ & \\
\hline \multicolumn{6}{|l|}{ Familia Strombidae } \\
\hline Strombus gigas & $\mathrm{x}$ & & & $\mathrm{x}$ & \\
\hline \multicolumn{6}{|l|}{ Familia Naticidae } \\
\hline Polinices hepaticus & $\mathrm{x}$ & & & $\mathrm{x}$ & \\
\hline Polinies lacteus & $\mathrm{x}$ & & & $\mathrm{x}$ & \\
\hline \multicolumn{6}{|l|}{ Familia Muricidae } \\
\hline Stramonita biserialis & & $\mathrm{x}$ & & $\mathrm{x}$ & \\
\hline \multicolumn{6}{|l|}{ Familia Columbellidae } \\
\hline Columbella fuscata & & $\mathrm{x}$ & & $\mathrm{x}$ & \\
\hline Columbella major & & $\mathrm{x}$ & & $\mathrm{x}$ & \\
\hline Nitidella nítida & $\mathrm{x}$ & & & $\mathrm{x}$ & \\
\hline Familia Vasidae & & & & $\mathrm{x}$ & \\
\hline Turbinella anulata & $\mathrm{x}$ & & & $\mathrm{x}$ & \\
\hline \multicolumn{6}{|l|}{ Familia Olividae } \\
\hline Oliva incrassata & & $\mathrm{x}$ & & $\mathrm{x}$ & \\
\hline
\end{tabular}


Clio Arqueológica 2021, V36N2, p. 297-318, ARELLANO; MOLINA; MENDEZ

DOI: 10.51359/2448-2331.2021.252351

\begin{tabular}{|c|c|c|c|c|c|}
\hline Oliva julieta & & $\mathrm{x}$ & & $\mathrm{x}$ & \\
\hline Oliva reticularis & $\mathrm{x}$ & & & $\mathrm{x}$ & \\
\hline Oliva sayana & $\mathrm{x}$ & & & $\mathrm{x}$ & \\
\hline Oliva scripta & $\mathrm{x}$ & & & $\mathrm{x}$ & \\
\hline Olika spendidula & & $\mathrm{x}$ & & $\mathrm{x}$ & \\
\hline Oliva)volutella & & $\mathrm{x}$ & & $\mathrm{x}$ & \\
\hline Olivella zonalis & & $\mathrm{x}$ & & $\mathrm{x}$ & \\
\hline Agaronia testacea & & $\mathrm{x}$ & & $\mathrm{x}$ & \\
\hline \multicolumn{6}{|l|}{ Clase Bivalvia } \\
\hline \multicolumn{6}{|l|}{ Familia Pteriidae } \\
\hline Pinetada mazatlanica & & $\mathrm{x}$ & & $\mathrm{x}$ & \\
\hline \multicolumn{6}{|l|}{ Familia Spondylidae } \\
\hline Spondylus/calcifer & & $\mathrm{x}$ & & $\mathrm{x}$ & \\
\hline \multicolumn{6}{|l|}{ Familia Unionidae } \\
\hline Nephronaias aztecorum & & & & & $\mathrm{x}$ \\
\hline $\begin{array}{l}\text { Megalonaias nikliniana } \\
\text { digitata }\end{array}$ & & & & & $\mathrm{x}$ \\
\hline \multicolumn{6}{|l|}{ Familia Chamidae } \\
\hline Chama echinata & & $\mathrm{x}$ & & $\mathrm{x}$ & \\
\hline \multicolumn{6}{|l|}{ Familia Tellinidae } \\
\hline Arcopagia fausta & $\mathrm{x}$ & & & $\mathrm{x}$ & \\
\hline \multicolumn{6}{|l|}{ Familia Veneridae } \\
\hline Chione sp. & & & $\mathrm{x}$ & $\mathrm{x}$ & \\
\hline \multicolumn{6}{|l|}{ Subtotal } \\
\hline Polyplacophora & & 1 & & 1 & \\
\hline Gastropoda & 8 & 10 & 1 & 5 & \\
\hline Bivalvia & 1 & 3 & 1 & 20 & 2 \\
\hline TOTAL & 9 & 14 & 2 & 26 & 2 \\
\hline
\end{tabular}

Figura no. 3.- Tabla 1. Procedencia y hábitat de las especies de moluscos identificadas. Los 454 elementos recuperados se agruparon en tres categorías de acuerdo con su uso: ornamental, utilitario y votivo.

De la clase Polyplacophora se identificó una especie, de la Gastropoda 20 y de la Bivalvia cuatro especies y un género. De las 20 especies identificadas, cinco caracoles provienen de la provincia Caribeña (ocho caracoles y una almeja), 14 de 
la Panámica (un quitón, 10 gasterópodos y tres bivalvos), un caracol se distribuye en ambas costas y dos especies de almejas que viven en los ríos.

\section{USO ORNAMENTAL}

Incrustaciones: Dentro de este término se incluyeron los objetos ornamentales cuyas características morfológicas permiten suponer que fueron pegados o cosidos a algún otro material (SUÁREZ, 1977: 56).

\begin{tabular}{|l|l|l|l|l|l|}
\hline Ofrendas & 123 & 125 & 126 & 137 & Total \\
\hline Taxa identificados & & & & & \\
\hline Clase Gastropoda & & & & & \\
\hline Strombus gigas & & 13 & & & $\mathbf{1 3}$ \\
\hline Turbinella angulata & 3 & 1 & & & $\mathbf{4}$ \\
\hline Clase Bivalvia & & & & & \\
\hline Pinctada mazatlanica & & 49 & & & $\mathbf{4 9}$ \\
\hline Spondylus calcifer & & & 2 & & $\mathbf{2}$ \\
\hline Chione sp. & & 1 & & & $\mathbf{1}$ \\
\hline Bivalvo no identificable & 1 & & & & $\mathbf{1}$ \\
\hline Total & $\mathbf{4}$ & $\mathbf{6 4}$ & $\mathbf{2}$ & & $\mathbf{7 0}$ \\
\hline
\end{tabular}

Figura no. 4.- Tabla 2. Especies utilizadas en la manufactura de incrustaciones. Orejeras: Son elementos que se colocaban sobre la oreja para ornamentarla; podían insertarse en la oreja o bien suspenderse del lóbulo (SUÁREZ, 2004: 71).Se recobraron cuatro orejeras Epcololli en la Ofrenda 125, fueron manufacturadas con la valva de Pinctada mazatlanica (ZÚÑIGA, 2013). Pectorales: Comprenden aquellas piezas que, gracias a las representaciones pictográficas y escultóricas, sabemos que se utilizaban suspendidas sobre el pecho (VELÁZQUEZ, 1999: 70).

Se recuperaron un total de 70 incrustaciones de dos ofrendas, cuatro provienen de la Ofrenda 123, tres fueron manufacturadas con Turbinella angulata y una con la valva de una almeja no identificable. De la 125 se recuperaron 64, 13 fueron hechas con fragmentos del cuerpo de la espira de Strombus gigas, una de

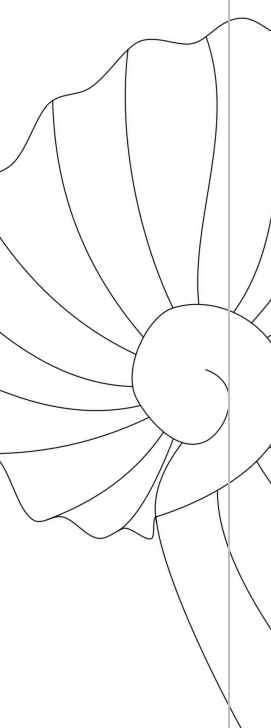


Turbinella angulata, 49 de Pinctadada mazatlanica y una de Chione sp., de la Ofrenda 126 tenemos dos de Spondylus calcifer (ZÚÑIGA 2013, 2014).

\begin{tabular}{|l|l|l|l|l|l|}
\hline Ofrendas & 123 & 125 & 126 & 137 & Total \\
\hline & & & & & \\
\hline Taxa identificados & & & & & \\
\hline Clase Gastropoda & & & & & \\
\hline Turbinella angulata & 1 & 2 & & & 3 \\
\hline Clase Biyalvia & & & & & \\
\hline Pinctada mazatlanica & 7 & 5 & & 2 & 14 \\
\hline $\begin{array}{l}\text { Megalonaias nikliniana } \\
\text { digitatd }\end{array}$ & & & & 2 & 2 \\
\hline Arcopdgia fausta & & & 1 & & 1 \\
\hline Total & 8 & 7 & 1 & 4 & 20 \\
\hline
\end{tabular}

Figura no. 5.- Tabla 3. Especies empleadas en la elaboración de pectorales.

Se exhumaron 20 pectorales en las cuatro ofrendas, de la Ofrenda 123 tenemos ocho, uno de ellos fue manufacturado con el gasterópodo Turbinella angulata y siete con la valva de Pinctada mazatlanica. De la Ofrenda 125 proceden siete, dos fueron elaborados con Turbinella angulata y cinco con Pintada mazatlanica. En la Ofrenda 126 se recuperó un pectoral manufacturado con la valva de Arcopagia fausta. Finalmente, de la Ofrenda 137 se obtuvieron 4, dos están hechos con la valva de Pintada mazatlanica y dos con la valva de la almeja de agua dulce Megalonaias nikliniana digitata (ZÚÑIGA, 2013; 2014; 2015). 
Pendientes: Son todas aquellas piezas ornamentales que presentan una o más perforaciones para ser suspendidas mediante un hilo o cordón y respecto a las cuales los objetos no guardan una simetría radial (SUÁREZ, 1977: 21).

\begin{tabular}{|l|l|l|l|l|l|}
\hline Ofrendas & 123 & 125 & 126 & 137 & Total \\
\hline Taxa identificados & & & & & \\
\hline Clase Gastropoda & & & & & \\
\hline Nerita scabricosta & & & 13 & & \\
\hline Neritina virginea & & 71 & & 17 & 88 \\
\hline Polinices hepaticus & 4 & & 5 & & 9 \\
\hline Polinices lacteus & & & 2 & & 2 \\
\hline Columbella fuscata & & 46 & & 5 & 51 \\
\hline Columbellamajor & & 7 & & & 7 \\
\hline Nitidella nítida & & 1 & & & 1 \\
\hline Oliva incrassat & & 8 & & & 8 \\
\hline Oliva julieta & & 1 & & & 1 \\
\hline Oliva reticularis & & & 5 & & 5 \\
\hline Oliva sayana & & & 22 & & 22 \\
\hline Oliva scripta & & & 6 & 3 & 9 \\
\hline Oliva spendidula & & 11 & & & 11 \\
\hline Oliva volutella & 10 & 24 & 34 & 16 & 84 \\
\hline Olivella zonalis & & 86 & & & 86 \\
\hline Agaronia testacea & & 2 & & & 2 \\
\hline Clase Bivalvia & & & & & \\
\hline Pinctada mazatlanica & 6 & 13 & & $2 ?$ & 21 \\
\hline Chama echinata & & 1 & & & 1 \\
\hline Total & 20 & 271 & 87 & 43 & 421 \\
\hline
\end{tabular}

Figura no. 6.- Tabla 4. Especies utilizadas en la manufactura de pendientes. 
De estos ornamentos se recobraron 421 de las cuatro ofrendas, de la 123 tenemos 20 piezas, 14 fueron hecha con gasterópodos: cuatro con Polinices hepaticus y 10 con Olivella volutella, con la valva de la madreperla (Pintada mazatlanica) fueron elaborados seis pendientes en forma de gota. De la Ofrenda 125 se recuperaron 271 pendientes, 257 corresponden a gasterópodos y 14 a bivalvos, en la Ofrenda 126 tenemos 87 todos manufacturados con la concha de gasterópodos; finalmente de la Ofrenda 137 se exhumaron 43 piezas, 42 fueron hechos con caracoles y dos con Pinctada mazartlanica (ZÚÑIGA, 2013; 2014 y 2015).

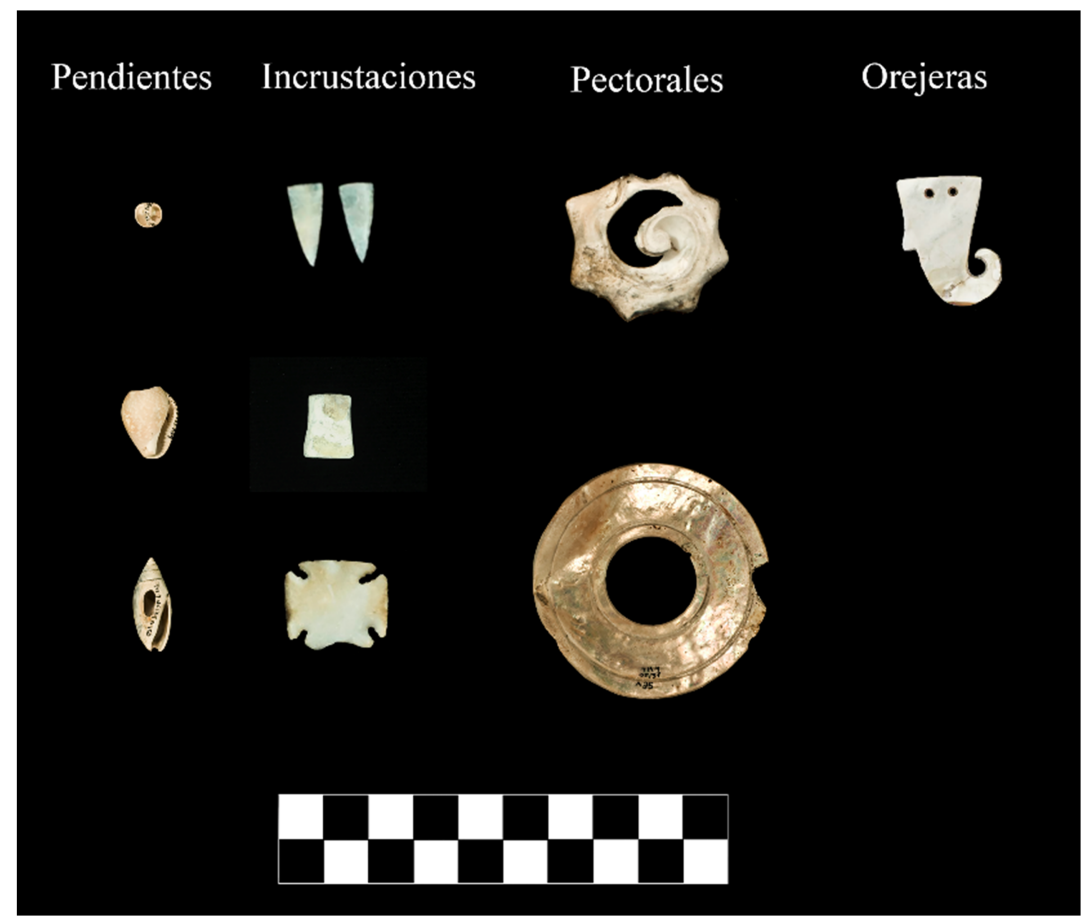

Figura 7. Objetos de concha clasificados como ornamentales, (Fotografía: Mirsa Islas, cortesía PTM-INAH).
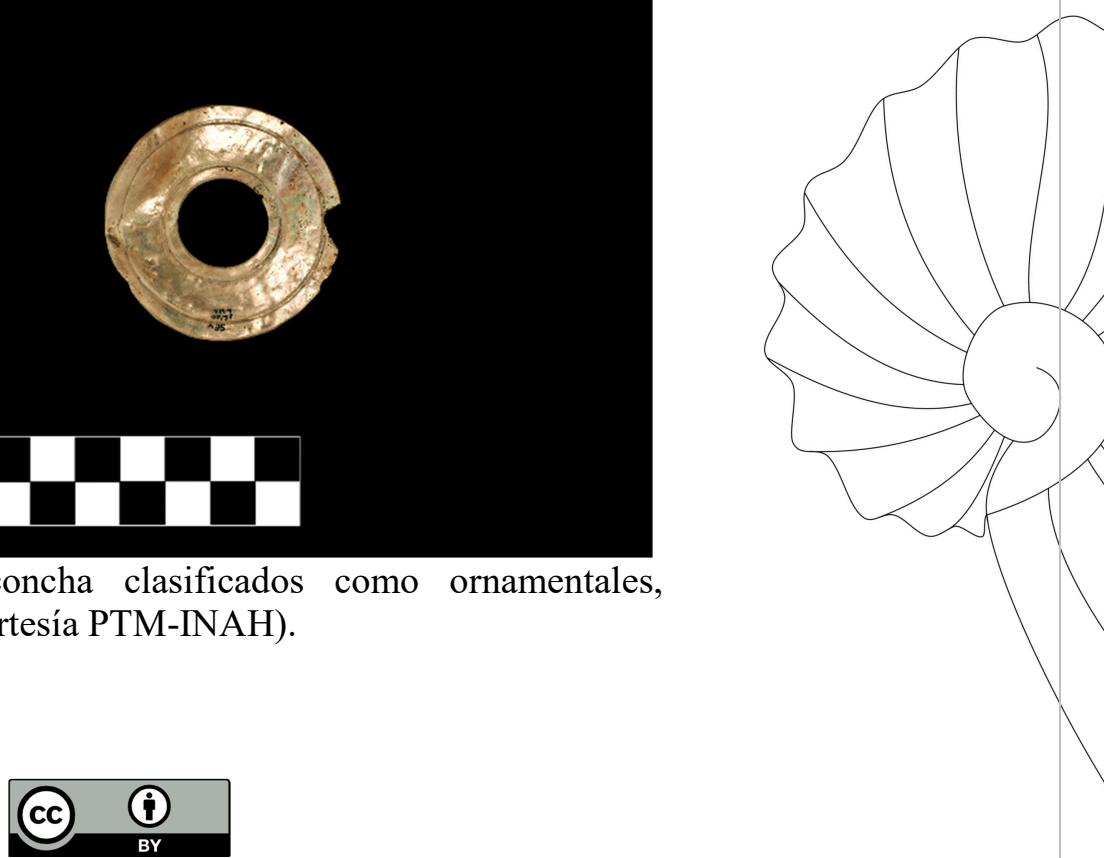


\section{USO UTILITARIO}

Lanzadardos: Se recuperaron cinco piezas procedentes de la Ofrenda 125, uno de ellos fue manufacturado con Strombus gigas y cuatro con Turbinella angulata, el pedestal de tres de los lanzadardos elaborados en la última especie fueron hechos con la valva de Pinctada mazatlanica (ZÚÑIGA, 2013).

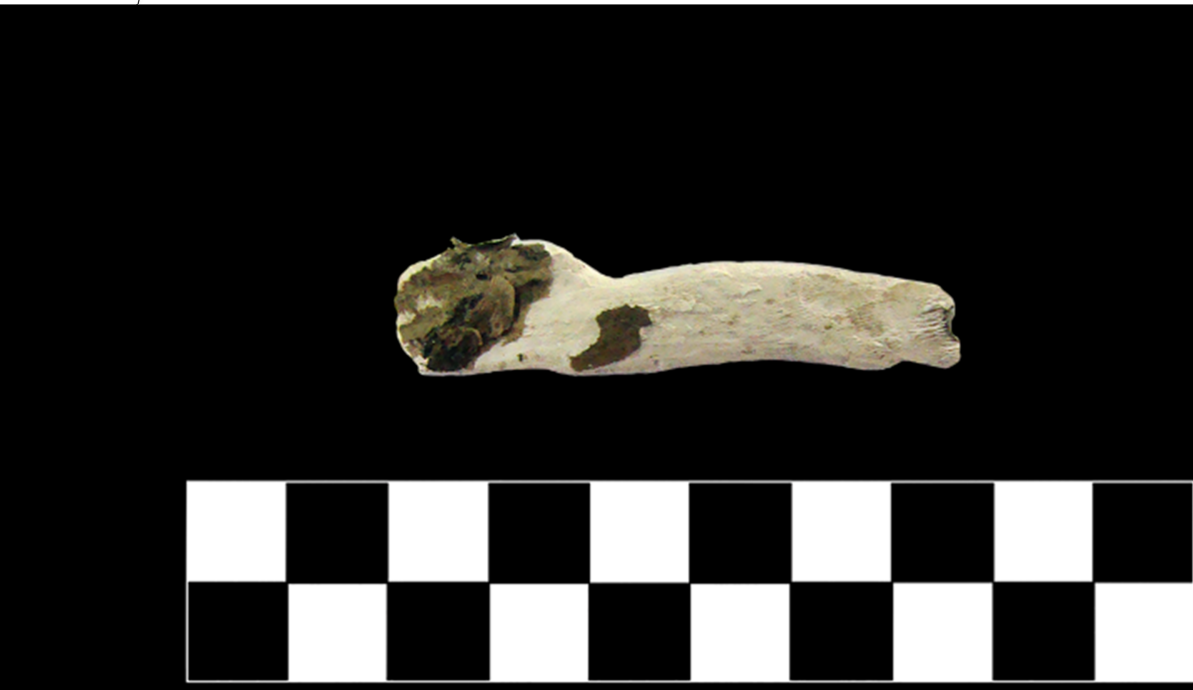

Figura 8. Lanzadardos de concha, (Fotografía: Mirsa Islas, cortesía PTM-INAH).

\section{USO VOTIVO}

Engloban a todas aquellas piezas manufacturadas con concha a las que no ha sido posible asignar otra función que la de servir como objeto de ofrendá (VELÁZQUEZ, 1999: 99). 
En esta categoría se identificaron 26 piezas en dos ofrendas. En la 125 se recuperaron 24, 10 fueron elaboradas con placas de "quitón" o "cucaracha de mar" (Chiton articulatus), sólo una de ellas tiene una perforación cónica en el centro, en las nueve restantes la superficie dorsal está desgastada y pulida. Del cuerpo de la espira de Stramonita biserialis, se identificó una pieza de forma oval. De la almeja de agua dulce Nephronaias aztecorum se recuperaron 11 piezas, algunas de ellas son de forma rectangular, otras triangulares y algunas más cuadrangulares. Con la valva de Chama echinata se hicieron dos, una de ellas es una valva pulida y con el contorno gastado, la otra pieza corresponde a un fragmento de charnela con el contorno desgastado. En la Ofrenda 126 se recuperaron dos piezas (ZÚÑIGA, 2013).

\begin{tabular}{|l|l|l|l|l|l|}
\hline Ofrendas & 123 & 125 & 126 & 137 & Total \\
\hline Taxa identificados & & & & & \\
\hline Clase Polyplacophora & & & & & \\
\hline Chiton articulatus & & 10 & & & 10 \\
\hline Clase Gastropoda & & & & & \\
\hline Stramonita biserialis & & 1 & & & 1 \\
\hline Clase Bivalvia & & & & & \\
\hline Nephronaias aztecorum & & 11 & 1 & & 12 \\
\hline Chama echinata & & 2 & & 2 \\
\hline Arcopagia fausta & & & 1 & & 1 \\
\hline Total & & 24 & 2 & & 26 \\
\hline
\end{tabular}

Figura no. 9.- Tabla 5. Especies utilizadas con fines votivos. 


\section{ICONOGRAFÍA DE LOS ARTEFACTOS}

En la Ofrenda 123 se localizaron 32 artefactos de concha que fueron utilizados como parte de los atavíos de once cuchillos-rostro de pedernal, estas aplicaciones simulaban los ojos y los dientes.

Tres cuchillos tenían un pectoral circular de concha como parte de sus atavíos, otros tres portaban pendientes en forma de gota, dos tenían objetos rectangulares acinturados de concha y cuatro contaban con pendientes automorfos del género Polinices, además de dichos ornamentos, también presentaban pequeñas representaciones de armamento: lanzadardos, y dardos miniatura de madera, así como mazos de obsidiana. Los elementos referidos, eran empleados por los guerreros, tal es el caso de pendientes de caracoles del género Polinices y los pectorales circulares que eran vistos como una insignia bélica que aludía a los guerreros estelares, además, formaban parte de los atributos de varias deidades entre ellas se encuentran Tezcatlipoca, Huitzilopochtli y Mictlantecuhtli (MATEOS, 1993: 78, 270).

Por otra parte, tenemos los pendientes de concha en forma de gota, elemento con simbolismo solar usado por deidades asociadas con la música, las flores, el juego y la danza, entre ellas Xochipilli, Tlahuizcalpantecuhtli, Ixtliltzin, Huehuecóyotl y Techálotl (VELÁZQUEZ, 2000: 64, 152-153). Los cuchillos que los portaban también tenían dardos miniatura de madera asociados.

\section{i}

(1)


En este depósito sobresalía la presencia de dos cuchillos rostro con base de copal, en los que, para simular los ojos, usaron aplicaciones de pedernal y de concha en forma de cruz de Malta (símbolo por excelencia del fuego y, a la vez, esquema gráfico del centro del universo y de los cuatro rumbos cardinales). Un cuchillo destaca del resto por ser el único del depósito de color café oscuro, fue colocado al centro del receptáculo asociado a la representación de una media luna manufacturada en concha y una placa que simula tres dientes romos, así como dos objetos rectangulares acinturados en forma de moño también elaborados en concha (AGUIRRE, 2019: 316).

Los elementos descritos anteriormente, son similares a los que porta el personaje 309 que está en el bulto mortuorio representado en la lámina 72 del Códice Magliabechiano (1996) esto y el hecho de que los cuchillos de esta ofrenda se hallaban asociados a restos óseos humanos que fueron cremados, podrían indicar que en este depósito se estaba haciendo alusión a la representación de un ritual funerario de guerreros de alto rango, donde el cuchillo de color café probablemente representaba al personaje principal.

La Ofrenda 125 se componía de poco más de 4000 elementos, entre los que recuperamos 371 artefactos de concha, 13 de ellos son pendientes en forma de gota, 64 son incrustaciones, cinco son lanzadardos, siete pectorales y 271 pendientes de concha. En la manufactura de estas piezas se utilizaron 17 especies, un género de bivalvo y una almeja no identificable.

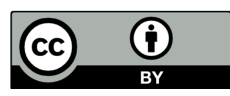


Estos elementos estaban asociados a 18 cuchillos efigie de pedernal y a los esqueletos de una loba mexicana (Canis lupus baileyi) y de un águila real (Aquila chrysaetos)

Uno de los cuchillos de pedernal que fue depositado, estaba vestido como el dios del viento Ehécatl-Quetzalcóatl, tenía el joyel del viento y las orejeras en forma de voluta elaborados en concha, así como un sartal de caracoles del género Olivella zonalis, el cetro curvo de obsidiana, la representación de un punzón de hueso hecho en lámina de oro, $\mathrm{y}$ un pendiente de piedra verde en forma de cabeza de pato -animal que es el nahualli de esta divinidad (CHÁVEZ et al. 2010: 73-75). Además, portaba un lanzadardos y dardos de madera.

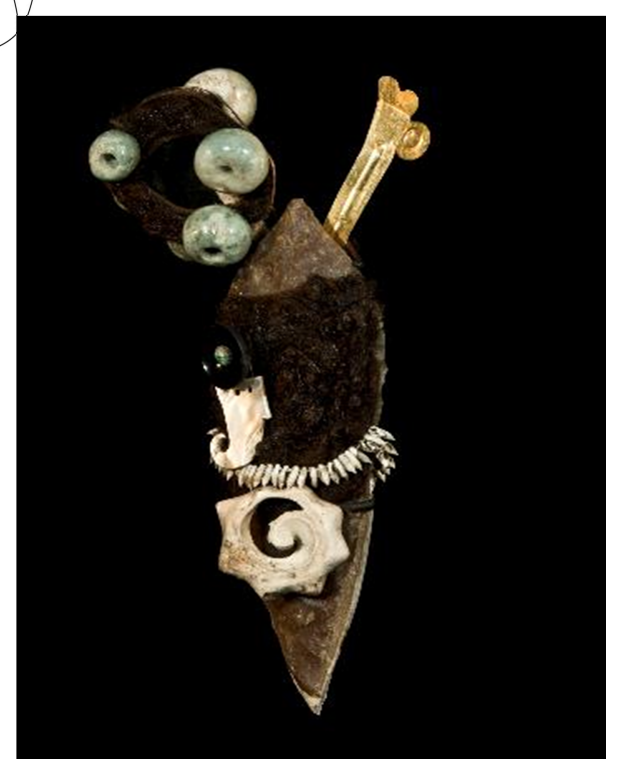

Figura 10. Representación del dios del viento Ehécatl-Quetzálcoatl, (Fotografia: Mirsa Islas, Cortesía PTM-INAH).

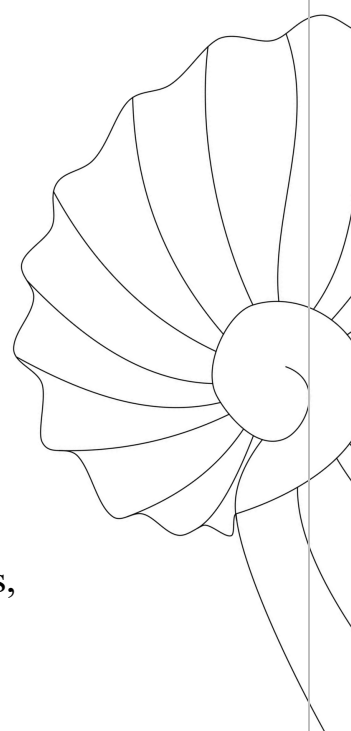



De los siete cuchillos restantes, tres portaban pectorales circulares de concha, dos tenían sartales elaborados con caracoles del género Neritina, otros dos cuchillos estaban ataviados con pendientes en forma de gota, uno de ellos tenía un pectoral circular y el otro un lanzadardos también de concha. Tenían además otros tipos de armamento como puntas de proyectil de pedernal y dardos de madera; así como otros ornamentos utilizados por los guerreros y personajes de la élite tales como pendientes de piedra verde y cascabeles de cobre. Hay que señalar que a todos ellos les colocaron aplicaciones de concha para simular ojos y dientes AGUIRRE y CHÁVEZ, 2010).

Los cuchillos estaban asociados a los esqueletos de dos águilas reales (Aquila chrysaetos), símbolos solares que se encontraban en la parte oeste del depósito. Ambas, portaban un pectoral circular, uno de ellos elaborado en concha y el otro en madera. Las dos aves estaban orientadas hacia el poniente región por donde desciende el sol al inframundo, por lo que en su calidad de guerreros estarían simbólicamente acompañando a este astro en su recorrido diurno desde que emerge de la región de los muertos hasta el cenit (AGUIRRE, 2019: 320-324).

Por otra parte, en el fondo del depósito colocaron el esqueleto de una loba mexicana (Canis lupus baileyi), estaba cubierta por miles de materiales marinos, conchas, caracoles peces, etc. Fue ataviada como un personaje de la élite, portaba un cinturón elaborado con pendientes manufacturados con caracoles del género Oliva, elementos empleados por deidades de carácter terrestre y asociados con la

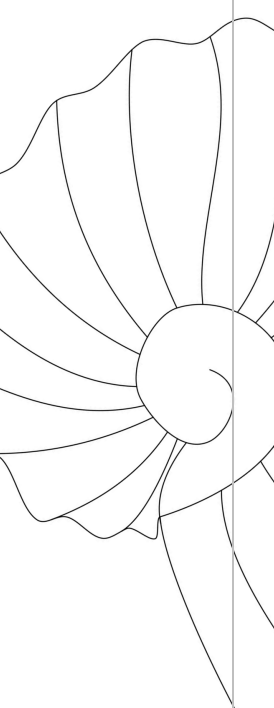


muerte, como las diosas Tzitzimime y el dios del inframundo Mictlantecuhtli (VELÁZQUEZ, 2000: 182). También tenía unas orejeras de madera cubiertas por pequeños mosaicos de turquesa, un collar elaborado con cuentas de piedra verde, y ajorcas de cascabeles de oro en las extremidades posteriores. El simbolismo de este animal entre los mexicas era similar al del coyote, ambos en su calidad de fieras eran ejemplo y símbolo de los guerreros. El lobo, además, simbolizaba el cielo nocturno y era portador de la noche (SELER, 2008: 64). Lo que concuerda con el hecho de que este animal haya sido depositado dentro de la ofrenda en el nivel que consideramos representaba al inframundo.

Acompañando al esqueleto de este animal encontramos otros 10 cuchillos de pedernal ataviados con artefactos de concha, representando a guerreros con diferentes tipos de armamento: siete cuchillos portaban pendientes de concha en forma de gota, igual que los cuchillos que se encontraban en la representación del nivel celeste, cuatro de ellos, además, tenían pectorales circulares de concha, cuentas de caracoles del género Neritina y lanzadardos de concha, así como pendientes en forma de moño y dardos de madera, también tenían puntas de proyectil de pedernal y la representación de pequeñas rodelas elaboradas conn fibras vegetales, seis de los cuchillos contaban con mazos de obsidiana, y con sartales de cascabeles de cobre. Todos ellos fueron cubiertos por una capa con cientos de organismos marinos, simulando estar en el inframundo de naturaleza acuática.

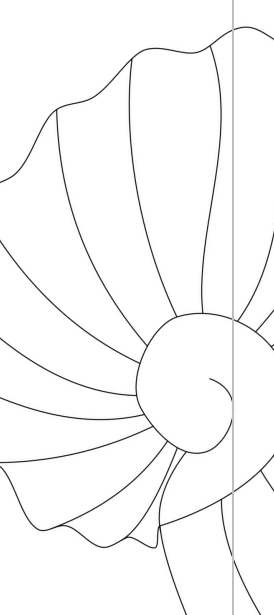


Considerando las descripciones anteriores, los cuchillos que portaban artefactos de concha de diversa índole, fueron colocados en este depósito para escenificar una batalla entre las fuerzas de la oscuridad y de la noche sobre el Sol; es decir, el descenso del astro, por el poniente y hacia el inframundo, pues como ya indicamos, la mayoría de ellos se encontraban armados. Como en el depósito antes mencionado, en esta ofrenda estarían varios cuchillos y animales ataviados representando a un grupo de guerreros de diferentes rangos -cuya jerarquía se vería reflejada por el tipo de insignias utilizada- encabezados por Quetzalcóatl.

La Ofrenda 126 es uno de los más grandes depósitos que se han localizado hasta el momento en las excavaciones del Templo Mayor de Tenochtitlan, se encontraba bajo el monolito de la diosa Tlaltecuhtli y estaba contenida en una caja de sillares de andesita de lamprobolita. Este depósito estaba conformado por más de 13000 elementos, en su mayoría de origen marino y restos óseos de fauna. En ella se encontraron 25 artefactos elaborados en conchas y caracoles, formaban parte del ajuar de 9 cuchillos de pedernal ataviados.

Se pudo determinar que ocho de los cuchillos contaban con pequeñas máscaras de madera que les fueron colocadas como sustituto del rostro, representando a personajes muertos (tienen los ojos cerrados y la boca entreabierta. En la plástíca mexica esta es la convención para representar a los muertos. Todos tenían anillos (anáhuatl), miniatura de concha, así como dardos y lanzadardos de madera. Uno de ellos, además, contaba con cuatro pendientes elaborados con caracoles Polinices.

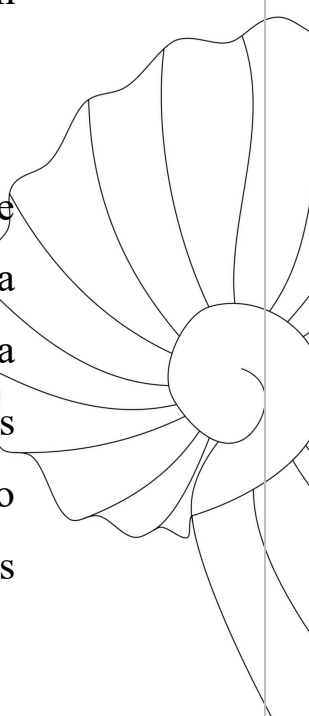


Hay que mencionar que otro de los cuchillos portaba un pectoral ehecacózcatl de concha, un sartal de 12 caracoles Olivella y un cetro curvo ehecatopilli de obsidiana. Esto nos permite definirlo como una representación de la deidad mexica del viento, Ehécatl-Quetzalcóatl.

En este depósito se estaba representado un cosmograma. En un primer nivel se encontraban 15 cuchillos de pedernal ataviados, la mayoría de ellos orientados hacia el este. Todos tenían grandes bases de copal en forma de cono truncado, tanto por esto, como porque se encontraban al sur y al norte del cartílago rostral de un pez sierra (el cual dentro de la cosmovisión mexica simbolizaba la superficie terrestre), dichos cuchillos con sus bases pudieron estar fungiendo como pequeñas representaciones de cerros deificados, es decir, una simulación a escala de la orografía de la Cuenca de México. Abajo del cartílago rostral de pez sierra se encontraban cientos de elementos marinos, marcando la separación de la representación del nivel terrestre con el mar. Bajo el nivel acuático, fueron depositados miles de restos óseos de fauna correspondientes a lobos, jaguares, linces, pumas, águilas, gavilanes, halcones, búhos y serpientes de cascabel, entre otros. Es conocido que en el inframundo de naturaleza acuática se encuentra el Mictlan, segmento del axis mundi, lugar cósmico en donde se produce el ciclo vida/muerte (LÓPEZ y LÓPEZ, 2009: 107).

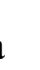


Los depósitos referidos hacen alusión a conceptos sobre la tierra, la fertilidad y la muerte. Entre muchas de sus funciones las ofrendas generan a la fecundidad de la tierra y a la producción de la lluvia, motivo por el que los materiales marinos, como las conchas y los caracoles, abundan entre los dones. Cada objeto por sí mismo rebosa de una multitud de significados.

La Ofrenda 137 estaba conformada por 1211 elementos pertenecientes principalmente a conchas y caracoles. En ella encontramos 47 artefactos elaborados con conchas y caracoles asociados a 8 cuchillos efigie de pedernal.

De los cuchillos mencionados, pudimos determinar que la mayoría eran 314 representaciones de guerreros que se encontraban en el inframundo. Cinco cuchillos portaban pectorales circulares de concha. Como el caso de la ofrenda 126, tres de ellos tenían máscaras antropomorfas simulando personajes muertos, tienen los ojos cerrados y la boca semiabierta, dos contaban además con dardos de madera.

Por otra parte, localizamos otros dos cuchillos que portaban pendientes caracoles del género Olivella, elementos que se han identificado con Venus, en su forma de estrella vespertina, guerrero estelar que se encuentra en su viaje de muerte y resurrección por el inframundo; uno de ellos tenía en la superficie, cerca del extremo más ancho, un fragmento de madera muy deteriorado, de forma plana e irregular, que podría corresponder a los restos de una máscara como las antes

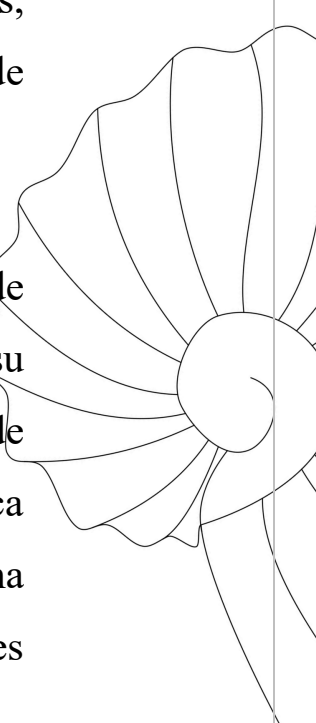




\section{Clio Arqueológica 2021, V36N2, p. 297-318, ARELLANO; MOLINA; MENDEZ}

DOI: 10.51359/2448-2331.2021.252351

mencionadas, los cuchillos también contaban con representaciones en miniatura de mazos de madera, uno de ellos además, tenía un dardo también de madera y una punta de proyectil de pedernal.

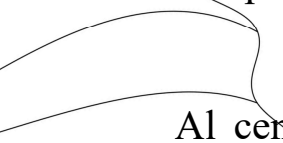

Al centro de la ofrenda se encontraba un cuchillo de pedernal blanco, que fue vestido de forma más sencilla, en la parte superior descansaba un pectoral circular elaborado con una valva de Pinctada mazatlanica.
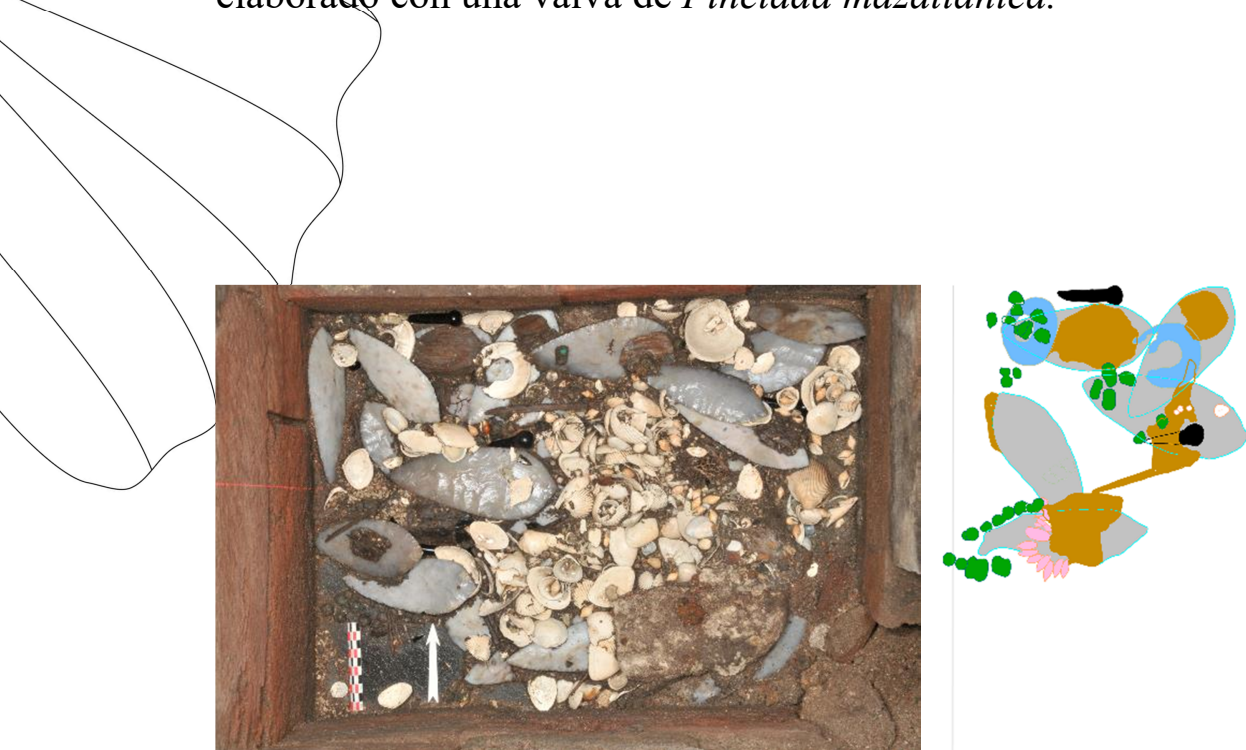

Figura 11. Los cuchillos de la Ofrenda 137 portaban Pectorales y pendientes de concha. a) Nivel 2 de excavación y b) dibujo de los cuchillos con sus ornamentos (dibujo de Israel Elizalde, cortesía PTM.INAH).

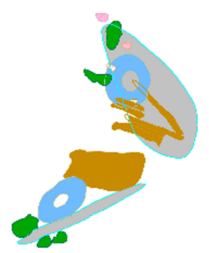

Al igual que en la Ofrenda 123, es sobresaliente el caso de un cuchillo de pedernal café oscuro, depositado cerca del eje central de la ofrenda. Sobre su superficie se 
encontró un fragmento de una posible máscara de madera. También tenía asociados 10 pendientes de caracoles del género Olivella. Todos los cuchillos mencionados se encontraban rematados por cascabeles de cobre en la porción proximal. Cabe señalar que, en todos los depósitos mencionados, prevalece la representación de guerreros que portan ornamentos de concha, lo que evidencia lo valioso que era el empleo de este material como parte de los dones ofrendados.

\section{CONCLUSIONES}

El usq de herramientas como el registro digital facilita el entendimiento y estudio de las ofrendas del Templo Mayor de Tenochtitlan y de los dones que en ellas se depositaron. Los programas que se emplearon en esta investigación ayudaron a determinar las asociaciones entre los elementos. Así pudimos determinar con cuales artefactos de concha y de otras materias primas fueron ataviados los cuchillos de pedernal, el esqueleto de un águila real y de una loba mexicana. Lo que nos permitió entender el significado que guardaban entre sí en los depósitos.

La identificación biológica de los artefactos de concha fue fundamental para conocer las especies que se utilizaron en la elaboración de cada tipo de artefacto, ahora sabemos que los artefactos fueron seleccionados por su forma y tamaño, en la manufactura de los pendientes se emplearon sólo ejemplares pequeños de caracoles Neritina y Olivella. Y en la confección de los pectorales principalmente se utilizó la Pictada mazatlanica. 


\section{Clio Arqueológica 2021, V36N2, p. 297-318, ARELLANO; MOLINA; MENDEZ DOI: $10.51359 / 2448-2331.2021 .252351$}

En suma, la integración de la biología, la arqueología y la iconografía dan como resultado una mejor interpretación de los contextos rituales, ayudándonos a entender la selección, procedencia y función de los organismos marinos y de agua dulce.

\section{REFERENCIAS BIBLIOGRAFÍCAS}

AGUIRRE, A. 2019 "Los cuchillos de pedernal ataviados de las ofrendas a Tlaltecuhtli”, Al pie del Templo Mayor de Tenochtitlan. Estudios en honor de Eduardo Matos Moctezuma, L. López y X. Chávez (coords.), México, El Colegio Nacional, v. 2 p. 313337.

AGUIRRE, A. y X. CHÁvEZ. 2010. Informe de la Operación 3. México: Archivo del Proyecto Templo Mayor.

CHÁveZ, X., A. AGUIRRE, A. MIRAMONTES y E. ROBLES. 2010. "Los cuchillos ataviados de la Ofrenda 125. Templo Mayor de Tenochtitlan", Arqueología Mexicana, n. 103 , p. $70-75$.

CHÁVEZ, X. Á. GONZÁLEZ, N. VALENTÍN y J. M. GARCÍA, Osteoarqueología de campo aplicada al análisis del uso ritual de la fauna: el caso de la Ofrenda 126 del Templo Mayor de Tenochtitlán. Estudios de Antropología Biológica. México: UNAM, n. 15, 2011, p. 117-137.

CÓDICE MAGLIABECHIANO. 1996. Edición facsimilar, México: Fondo de Cultura Económica/ Akademische Druck-und Verlagsanstalt.

DEHOUVE, D. 2013. "El depósito ritual: un ritual figurativo", Convocar a los Dioses": Ofrendas mesoamericanas, Johanna B. (coord.), México: Consejo Nacional para la Cultura y las Artes, p. 605-638.

EFRENOV, I. A. Taphonomy: New Branch of Paleontology. Pan-American Geologist. Moscu: v. 74, 1940, p. 81-93.

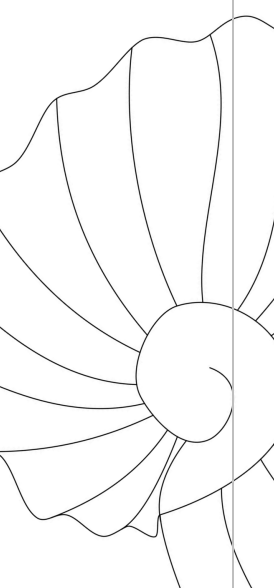


GARCÍA, J. M., D. MATADAMAS, I. ELIZALDE y A. GONZÁLEZ. 2014. Informe de la operación 11 y la ofrenda 137. México: Archivo del Proyecto Templo Mayor.

GIFFORD, D. Taphonomic and Paleoecology: a Critical Review of Archaeology's Sister Disciplines. en Michael B. Schiffer (ed.), Advances in Archaeological Method and Theory. Tucson: Academic Press, v. 4, 1981, p. 365-438.

GONZÁlEZ, A., X. CHÁVEZ, J., GARCÍA y B. ZÚÑIGA. 2012. Informe de la exploración de la Ofrenda 126. México: Archivo del Proyecto Templo Mayor.

KLEIN, C. 1984. “¿Dioses de la lluvia o sacerdotes ofrendadores del fuego? Un estudio sociopolítico de algunas representaciones mexicas del dios Tláloc", Estudios de Cultura Náhuratl, v. 17, p. 3-50.

LÓPEZ, A. y L. LÓPEZ. 2009. Monte Sagrado-Templo Mayor. El cerro y la pirámide en la tradición religiosa mesoamericana, México: Instituto Nacional de Antropología e Historia, Universidad Nacional Autónoma de México.

LÓPEZ, L. 1993. Las ofrendas del Templo Mayor de Tenochtitlan, México: Instituto Nacional de Antropología e Historia.

LÓPEZ, L. y X. CHÁVEZ. 2010. "Al pie del Templo Mayor: excavaciones en busca de los soberanos mexicas”, Moctezuma II. Tiempo y Destino de un Gobernante. López L. y Mcewan (coords.), México: Instituto Nacional de Antropología e Historia, p. 294-325.

LÓPEZ, L., X. CHÁVEZ, B. ZÚÑIGA, A. AGUIRRE y N. VALENTÍN. 2012. "Un portal al inframundo. Ofrendas de animales sepultadas al pie del Templo Mayor de Tenochtitlan”, Estudios de Cultura Náhuatl, v. 44, p. 9-40.

LYMAN 2010 "What Taphonomy Is, What it Isn't, and Why Taphonomists Should Care About the Difference", Journal of Taphonomy, Madrid: v. 8 2010, p. 1-16.

LYMAN, L. 1994. Vertebrate Taphonomy. Cambridge: Cambridge University Press, 1994.

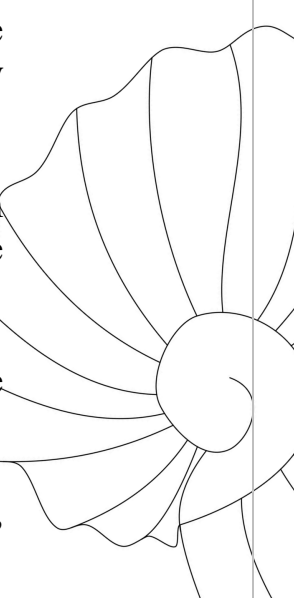




\section{Clio Arqueológica 2021, V36N2, p. 297-318, ARELLANO; MOLINA; MENDEZ} DOI: $10.51359 / 2448-2331.2021 .252351$

MATEOS, S. 1993. Los dioses creadores, México: Secretaría de Hacienda y Crédito Público.

PASCAL, C. y A. GONZÁLEZ. 2009. Informe de la exploración de la Operación 4 y las ofrendas 122, 123 y 124. México: Archivo del Proyecto Templo Mayor.

SELER, E. 2008. Las imágenes de animales en los manuscritos mexicanos y mayas. México. Casa Juan Pablos.

SUÁREZ, L. 1977. Tipología de los objetos prehispánicos de concha. México: Instituto Nacional de Antropología e Historia.

SUÁREZ, L. 2004. Conchas, caracoles y crónicas. México: Instituto Nacional de Antropología e Historia.

VELÁzQUEZ, A. 1999. Tipología de los objetos de concha del Templo Mayor de Tenochtitlan. México: Instituto Nacional de Antropología e Historia.

VELÁZQUEZ, A. 2000. El simbolismo de los objetos de concha encontrados en las frendas del Templo Mayor de Tenochtitlan. México: Instituto Nacional de Antropología e Historia.

ZÚÑIGA, B. 2013. Moluscos marinos asociados a la Ofrenda 125 del Templo Mayor de Tenochtitlan: Informe para el Consejo de Arqueología. México: Archivo del Proyecto Templo Mayor.

ZÚÑIGA, B. 2014. Identificación de los restos de moluscos recuperados en la Ofrenda 123. México: Archivo del Proyecto Templo Mayor.

ZÚÑIGA, B. 2015. Moluscos marinos asociados a la Ofrenda 137 del Templo Mayor de Tenochtitlan. México: Archivo del Proyecto Templo Mayor.

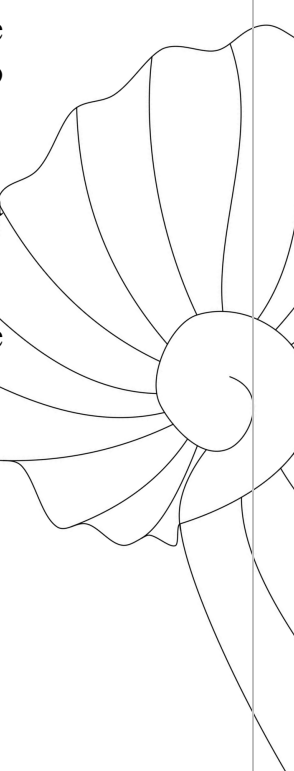

